\title{
Delocalized Photomechanical Effects of UV ns Laser Ablation on Polymer Substrates Captured by Optical Holography Workstation: An Overview on Experimental Result
}

\author{
Vivi Tornari \\ Foundation for Research and Technology-Hellas, Institute of Electronic Structure and Laser, N. Plastira 100, \\ Voutes, 73111 Heraklion, Crete, Greece \\ Correspondence should be addressed to Vivi Tornari; vivitor@iesl.forth.gr
}

Received 9 April 2014; Accepted 15 July 2014; Published 15 October 2014

Academic Editor: Zhipei Sun

Copyright (C) 2014 Vivi Tornari. This is an open access article distributed under the Creative Commons Attribution License, which permits unrestricted use, distribution, and reproduction in any medium, provided the original work is properly cited.

A brief overview of results from an experimental investigation performed on polymer materials to examine delocalized photomechanical effects generated due to UV laser ablation is provided. Delocalized structural modifications were observed in PMMA, PS by means of optical holographic interferometry. The integrity of samples was examined before and after irradiation in 193 and $248 \mathrm{~nm}(15 \mathrm{~ns})$ above and below ablation threshold, $F=0.1-1.0 \mathrm{~J} / \mathrm{cm}^{2}$. A value of structural continuity was initially determined for each sample by generation of a reference holographic image before irradiation. Microscopic discontinuities were intentionally induced to act as preexistent defects. Sequential holographic recording monitored the growth of morphological alterations according to the number of pulses in the neighbor as well as far from the preexistent discontinuities. The imposed alterations are visually observable at the whole extent of the irradiated sample in distances far off the ablation spot as local cracks and voids. The induced flaws cannot be classified as transient or instantly generated. Fracture follows a long-term emergence and deterioration pattern. Extensively fluctuated long-term effects were also observed in laser-assisted varnish removal of multilayered technical samples simulating Byzantine icons with decrease of effect duration and fluctuation being according to increase in sample homogeneity.

\section{Introduction}

Irradiation of solid organic material with pulsed ultraviolet laser light of intense power density leads to cold etching or ablative photodecomposition (APD) of the substrate. The APD effect located in the limited region of the laser spot is attributed to local photochemical mechanism causing discrete ejection of target material without altering the adjacent bulk which allows for controlled substrate processing conveniently suited for many applications. The mass ejection and the expansion of high-density plasma though induce a mechanical effect characterized by a recoil pressure and a shock wave propagating inside the solid. The photomechanical effects in contrast to the presented study are mainly reported in literature in concern to the physical processes in the ablated region as influence of pressure versus time.
Specifically, laser ablation using 193, $248 \mathrm{~nm}$ excimer laser radiation is commonly used [1-12] in microelectronic industry for etching of polymer substrates and in medical applications for controlled excision of tissue as well as in ophthalmology and in photorefractive surgery for restoring of myopia and has been proved to be a highly effective method in the restoration of painted artworks $[13,14]$. The material ablation mechanism caused upon the interaction of intense laser irradiation with a surface layer results in the explosive ejection of a significant amount of material from the specimen's surface at excimer laser fluence between $0.1 \mathrm{~J} / \mathrm{cm}^{2}$ and $0.5 \mathrm{~J} / \mathrm{cm}^{2}$ for varnish ablation, 0.2 and $1.5 \mathrm{~J} / \mathrm{cm}^{2}$ for polymer, and 0.5 and $1.9 \mathrm{~J} / \mathrm{cm}^{2}$ for biological tissue. The absorbed energy densities rise to few $\mathrm{kJ} / \mathrm{cm}^{3}$ and by being deposited within nanoseconds develop in the target 
substantial stress transients with amplitudes measured as high as $\sim 10^{5} \mathrm{~Pa}[15,16]$.

The implications thereupon of such high stresses on the target form the subject of this study. There have been evidences of damage in skin and cornea after ablation by ArFexcimer radiation at $193 \mathrm{~nm}[17,18]$. Also preliminary investigation on Byzantine icon models gives strong indications that formation and deterioration of defects related to the type of preexisting discontinuities were caused by the laser-assisted varnish removal at fluence from $0.29 \div 0.55 \mathrm{~J} / \mathrm{cm}^{2}$ [13-15]. Herein, we investigate the damage potential in polymer during and after irradiation at 193 and $248 \mathrm{~nm}$ to primarily understand the mechanisms governing the damage potential of photomechanical effect associated with the excimer laser ablation. The term damage potential is used in the context of morphological alterations induced in the structure instead of direct fracture. Polymers are chosen as model systems because their physical properties are better known.

For 193 and $248 \mathrm{~nm}$ irradiation and low radiant exposures, the stress wave is attributed to volume changes caused by photodecomposition, for higher radiant exposures where photoetching is observed to thermal expansion of the ablation plume and to plasma-mediated ablation. We use local continuous ablation creating one ablation spot in the target such as the photochemical products which remain localised within the spot. Operation in low pulse repetition rate $(1 \mathrm{~Hz})$ prevents interaction between the target formed plume/plasma and the incoming radiation minimising undesirable thermal effects. These parameters were kept to allow observation of the nonirradiated part of the target. In this paper, the study refers to observed effects outside the ablation spot.

In nondestructive material testing, holographic techniques have found extensive use for the characterization of both static and dynamic mechanical deformations and of the structural "condition" of a wide range of objects $[16,17]$. This wide applicability suggests the potential of the technique for the evaluation of plausible structural effects induced by UV laser ablation. Nevertheless, the use of holographic interferometry in the study of UV ablation has been quite limited and restricted mainly to the characterization of the plasma [18-20], an attempt at the characterization of the shock waves has been reported [12, 21-25]. It holds the advantage to allow instantaneous monitoring by full-field visualization in extended field-of-view which was exploited in the experiments shown.

\section{Experimental Description}

2.1. Experimental Setup. The experimental setup was designed for simultaneous photoablation of polymer substrates and holographic interferometric recording with the objective to acquire real-time holographic recording of the thermomechanical effects of UV laser ablation. The setup shown in Figure 1 is divided in two experimental pump-probe arms.

One is the arrangement for the UV ablation of the polymer targets consisting of the excimer laser with computer control for selection of parameters (repetition rate, voltage, etc.). The UV beam is directed to the sample by a series of mirrors and a focusing lens.

The second one is the arrangement for the holographic interferometric recording and reconstruction. The sample and the holographic setup stay in a dark room over a pneumatic isolation optical table. It consists of a twin beam off-axis holographic interferometer of which the one beam is diffusely transmitted through the phase object under investigation and the other beam having travelled across the same optical path serves as the reference beam. The two beams are interfering and their pattern of interference is recorded in the photosensitive medium. A CCD camera is positioned behind the hologram plane in order to digitalize the reconstructed image and send it, through a frame grabber, to an image processor.

The laser sources used are a Lambda Physik excimer laser (model LPX210) with different characteristics depending on the gas mixture, an ArF mixture in $193 \mathrm{~nm}$ wavelength with $23 \mathrm{~ns}$ nominal pulse duration and a $\mathrm{KrF}$ mixture in $248 \mathrm{~nm}$ wavelength with $34 \mathrm{~ns}$ nominal pulse duration. Also a continuous wave Helium-Neon laser (Uniphase, model $1125 \mathrm{P}$ ) class IIIb, with power output at $10 \mathrm{~mW}$ and wavelength of emitted radiation at $632 \mathrm{~nm}$, was used. The target material does not absorb the red light of the latter laser and it is used to record the holograms in transmission.

The set-up control devices are a workstation to start and stop the UV irradiation process and set the repetition rate; a joule-meter and an oscilloscope to measure the UV beam power on the object plane, after the losses due to the previous optical components; a power meter to measure the object beam, the reference beam, and the total intensities in the holographic setup; and a shutter and a chronometer, just after the Helium-Neon laser, to control the time exposure of the photosensitive plate and through the CCD.

2.2. Experimental Methodology. The recording geometry of the probing arm in the experiment makes use of high spatial resolutions $\left(f=\sin \theta / \lambda, \theta=45^{\circ}\right.$, and $\lambda=632 \mathrm{~nm}$ stands for $\mathrm{He}-\mathrm{Ne}$ ) in a two-beam off-axis transmission mode optical system (Figure 1). The one beam, termed probe or object beam, is diffusely transmitted through the sample forming the object wave

$$
U_{0}(x, y)=a_{0}(x, y) \exp \left[-i \phi_{0}(x, y)\right]
$$

to interfere with the reference wave

$$
\left[U_{R}(x, y)=a_{R}\right]
$$

in the recording plane, $(z=0)$. The polymer on suprasil window is firmly held on a holder in front of the photosensitive medium, at $z=0$, and before excimer irradiation the first hologram is recorded. The excimer laser is allowed to deliver the first pulse onto the polymer target and a second overlapping hologram is formed $U_{0}(x, y)=a(x, y) \exp \{-i[\phi(x, y)+$ $\Delta \phi(x, y)]\}$, where $\Delta \phi(x, y)$, the irradiation change, is induced in the object. The phase term $\Delta \phi$ is related to the physical quantity of interest, for which the particular application is 


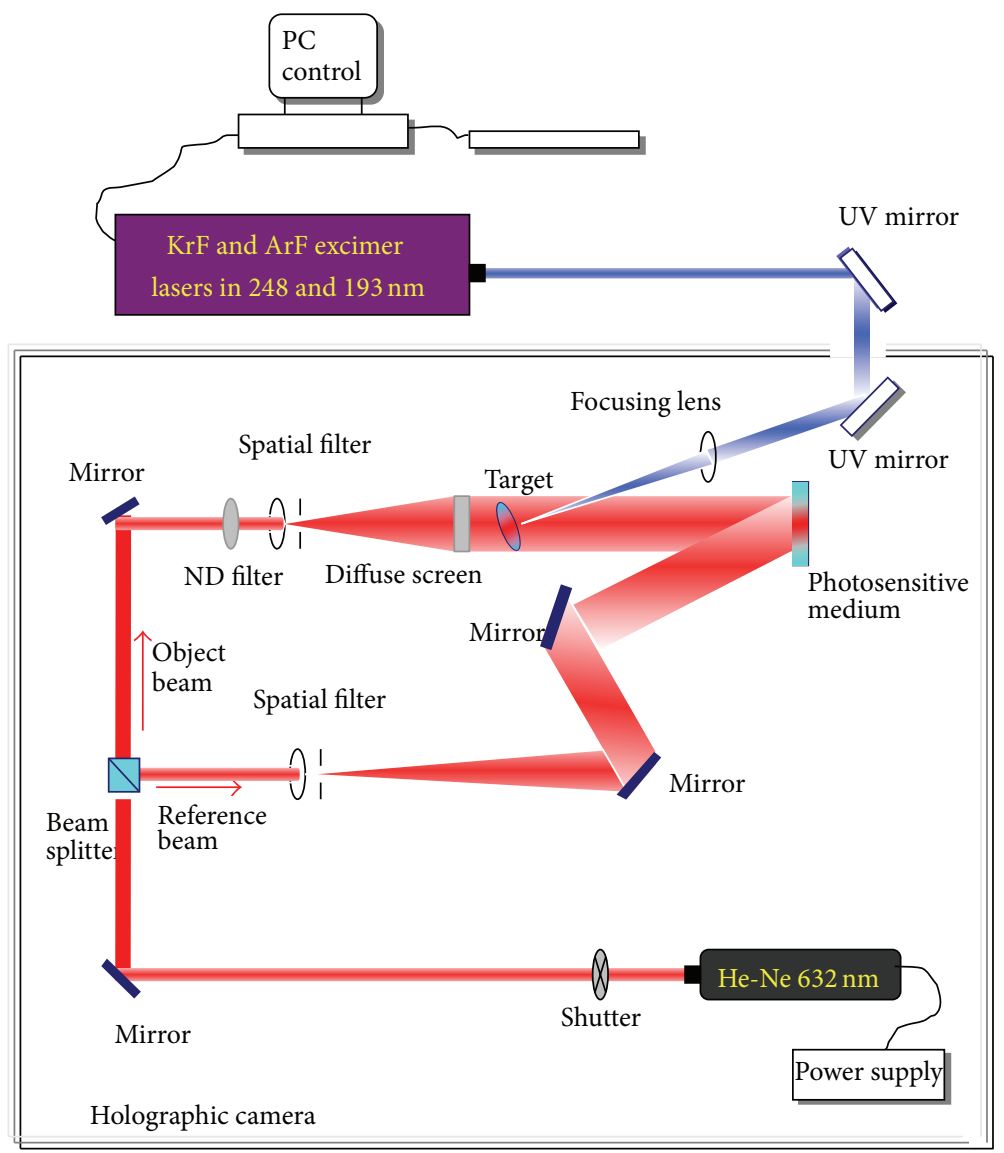

FIGURE 1: Drawing of the optical geometry used for online polymer ablation monitoring. Note that the UV beam is focused while the monitoring beam $(\mathrm{OB})$ is expanded to cover the whole diameter of the sample.

the refractive index change that is induced by the ablation of polymer material in the ablation spot. If the refractive index change equals the integral multiple of $\lambda$, the interference fringes are generated:

$$
\Delta \Phi=\int\left[n(x, y, z)-n_{0}\right] d z=N \lambda
$$

This implies that when the field has uniform irradiance an infinitely wide fringe results, $\Delta \phi(x, y)=0$. Before the second excimer pulse, a third hologram according to (1) and (2) is formed in a different position of the photosensitive medium and the excimer delivers the second pulse after which the forth hologram is recorded with $\Delta \phi_{2-3}(x, y)$. In this sequence, a holographic wavefront is getting modulated by each or number of pulses to record in the photosensitive medium potential modifications in every step of the ablation process in terms of phase changes:

$$
\sum \sum\left[\Delta \phi_{2-1}(x, y), \Delta \phi_{3-2}(x, y) \cdots \Delta \phi_{(n-1)-n \text {th }}(x, y)\right]
$$

A holographic diffuse object wave provides observation from different angles of view to pinpoint spatial coordinates displaced in unknown directions. The oblique observation allocates modifications within the range of uniform irradiance allowing detection of changes that are not identified by generation of fringes related to refractive index change. Typical case is the structural changes forming defects that can be spatially confined or extended but nonetheless without influencing the refractive index of the polymer. At the end of irradiating-recording procedure, a continuous monitoring of ablation process is visualized with each subsequent hologram reconstructing distinctively the evolution of induced structural alterations during the ablation. Since in a holographic interferometer only changes in path length between exposures are displayed $\Phi_{2}(x, y)-\Phi_{1}(x, y)$, a fixed structural change present in both exposures is cancelled allowing only new or unstable changes in each recording. Spatial phase encoding and recording of induced structure/mechanical displacements of generated alterations could lead to monitoring and interactive self-operating control of ablation procedures.

2.3. Experimental Procedure and Sample Preparation. The polymer used in the experiments was pure and blend of PMMA/PS with varying concentrations layered at $\approx 50$ $120 \mu \mathrm{m}$ thicknesses on suprasil window of $5 \mathrm{~cm}$ diameter irradiated in air with excimer laser pulses at 193 and $248 \mathrm{~nm}$. 
The irradiation spot was $\approx 0.2 \times 0.8 \mathrm{~mm}$ and the fluence was $0.4-0.6 \mathrm{~J} / \mathrm{cm}^{2}$.

Samples preparation was performed on chemistry laboratory in ambient conditions in systematic steps. Control procedures are thus envisaged to confirm assigned deformation value due only to laser irradiation. Firstly, for every polymeric material to be investigated, two samples were made with identical procedure and at the same time. While one was irradiated, the other was kept protected as reference sample producing evidence of structural degradation due to inherent residual stresses against the degradation of the ablated sample. Therefore, the irradiated sample was compared to the reference intact one with identified natural changes. In this manner, the nonirradiated reference sample was the indicator of residual tensions of the material independently of the UV irradiation process.

Secondly, most of the samples possessed preexistent defects built during the sample preparation process. Hence, a hologram of the sample was recorded before the starting of UV irradiation in order to have a reference localization map of the preexistent defects. The experimental methodology to record holographic interferograms is summarized in Section 2.2.

Thirdly, an optical microscope (Nikon eclipse ME600) was also implemented to pinpoint the preexistent defects and observe selected areas of the sample of a spatial resolution $\approx 100$ microns depending on the lens magnification. The selected areas were chosen either in the center of the sample where the ablation would take place, in the vicinity, or in the further far corners. Thus, the surface morphology before and after the irradiation process could be compared. The optical microscope does not allow a full-field view of the spatial phenomenon as is acquired with the holography procedure emphasizing more local morphological changes with higher magnification, thus complementing the comparison process in many cases.

Finally, SEM was also implemented to distinguish detected defect morphologies. It should be highlighted here that it is not the optical damage caused by the pulse in the ablation spot that was of interest in this study but the delocalized effect on surface morphology in the extent of the sample.

\subsubsection{Preparation of Polymeric Layers on Suprasil Window.} The sample preparation was realized diluting the pure polymer or the blend powder with a proper weight percentage in a stoichiometric quantity of dichloromethane. After a careful cleaning of the suprasil window, it was positioned on a perfectly horizontal plane and a known quantity of the polymeric solution was spread on it; this quantity, together with the dilution level of the solution, determined the thickness of the polymer layer. Then, the sample was covered in order to slow down the natural dichloromethane evaporation, otherwise, too fast and risky for a good preparation. When the solution was dry, the sample was ready to be irradiated.

2.3.2. Preparation of Solid Polymers. The dilution for the solid polymers was realized under the same procedure as above.
Then, it was poured in a square base mould and covered until the material was completely dry. No reference sample was prepared because the solid polymers do not present natural degradation, to compare with the one created by the excimer laser.

\section{Results}

The experiments were performed according to following parameters.

Type of Sample. The influence of interface and adhesion strength was investigated with experiments performed either on samples formed by a film of material on suprasil window or on pure solid samples.

Laser Wavelength. The influence of wavelength with a material changing the characteristics of the process in terms of absorption coefficient was investigated for an ArF $193 \mathrm{~nm}$ and a $\mathrm{KrF} 248 \mathrm{~nm}$.

Molecular Weight. The influence of molecular weight in mechanical resistance was investigated by construction of polymer samples with different molecular weights (PMMA: $\mathrm{MW}=1.900 \mathrm{u}$ and $\mathrm{MW}=100.000 \mathrm{u}$ and PS: $\mathrm{MW}=1.180 \mathrm{u}$ and $\mathrm{MW}=280.000 \mathrm{u}$ ).

Direction of Irradiation. A critical factor in many applications is the position of absorbing chromophores in the ablated material; hence, the direction of irradiation was tested forward to the surface of the sample and backward through the substrate where for both cases it was almost perpendicular to the sample.

3.1. Study on Neat Polymer at $193 \mathrm{~nm}$. The absorption coefficient of PMMA in $193 \mathrm{~nm}\left(\alpha=1 / 2.08 \times 10^{-4}\right)$ is considered relatively high compared to longer wavelengths in the UV range. Calculation suggests that the ratio of the etch depth of PMMA close to the ablation threshold over the light penetration depth is one order of magnitude larger in the case of irradiation at $193 \mathrm{~nm}$ than in the case of irradiation at $248 \mathrm{~nm}$ [26]. This derives from the etch depth/cm for PMMA in $193 \mathrm{~nm}$ at $F=100 \mathrm{~mJ} / \mathrm{cm}^{-2}$ which ranges from 0.10 to $0.15 \times 10^{-4}$ and penetration depth from 2.08 to 2.22 $\times 10^{-4}$ and the resulting ratio is 0.06 ; while for $248 \mathrm{~nm}$ etch depth $/ \mathrm{cm} 0.8 \times 10^{-4}$ at $F=800 \mathrm{~mJ} / \mathrm{cm}^{-2}$ and penetration depth $0.98 \times 10^{-2}$ resulting ratio is 0.008 . For the case of PS, an order of magnitude difference in 193 and $248 \mathrm{~nm}$ has been also calculated. It is thus anticipated that most of the photons energy is effectively consumed in material removal through photodecomposition. The efficient ejection of material is supposed to generate strong shock wave into the bulk material increasing the photomechanical influence.

Figure 2 illustrates the results of PMMA at $193 \mathrm{~nm}$. The experiments started on neat PMMA samples $(\mathrm{Mw}=$ $120.000 \mathrm{u}$ ) to establish the operational parameters first on well-defined homogeneous material. There was however low formation of defects of the order of $a_{\text {def }} / a_{\text {total }} \times 100=0.75 \%$ 

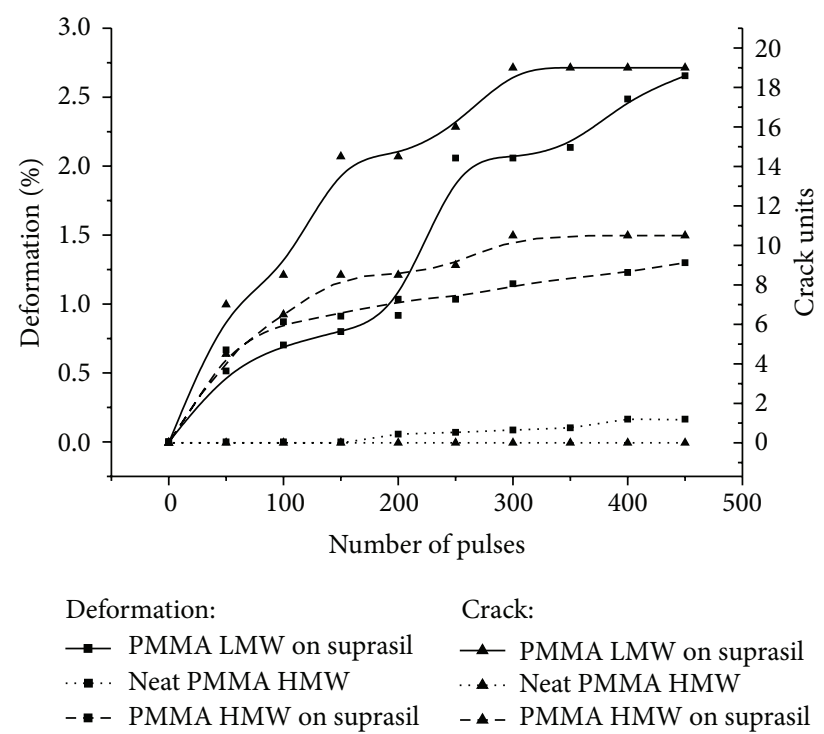

FIGURE 2: Defect formation versus number of pulses of neat PMMA at $193 \mathrm{~nm}$.

only after 400 pulses. Deformation equals the ratio derived by the area of deformation in $\mathrm{cm}^{2}$ to the total area of the substrate $\left(\mathrm{cm}^{2}\right)$. The resulted integer provides the degree of deformation. The defect morphology was dot-like expansions or separations of bulk material mainly in the regions with located preexistent discontinuities. The neat PMMA ( $\mathrm{Mw}$ $=120.000 \mathrm{u}$ ) was then spread as film on suprasil window and was irradiated under the same number of pulses. The defect formation was dot-like structures of $1 \%$ magnitude at 200 pulses instead of 400 for the solid sample. Another PMMA film on suprasil window was prepared with lower molecular weight $(\mathrm{Mw}=1900 \mathrm{u})$ and at 200 pulses the dotlike defect morphology was of the order of $0.9 \%$, and at 250 pulses it raised to $2 \%$ with additional generation of cracklike structures. Cracks were randomly spread and oriented all over the material and were measured over a minimum reference length of $0.4 \mathrm{~mm}$ unit for crack, where for the given sample calculated crack formation resulted in 19 unit cracks with total length $76 \mathrm{~mm}$. It should be also noted here that the results were acquired with $1 \mathrm{~Hz}$ repetition rate.

More experiments on neat film on window were performed with PS $(\mathrm{Mw}=1180 \mathrm{u})$ and distinct behaviour was exhibited. PS presented also both defect morphologies but compared to PMMA was exhibited in considerable smaller magnitude of deformation with $0.1 \%$ at 200 pulses rising after the 300 pulses at just $0.5 \%$ and 7 unit cracks (Figure 3). An explanation of this behaviour could be based on the different absorption coefficients of the two polymers. In $193 \mathrm{~nm}$, PS absorbs more effectively than PMMA penetrating less in depth of material bulk resulting in less influence in the overall bulk of material (schematic diagram at the end).

In the graph shown in Figure 2, it is clearly seen that the higher "defect formation" ("defect formation" is a term implied to present both "deformation and crack unit" in the graphs) of the PMMA sample of low $\mathrm{Mw}$ on window is in

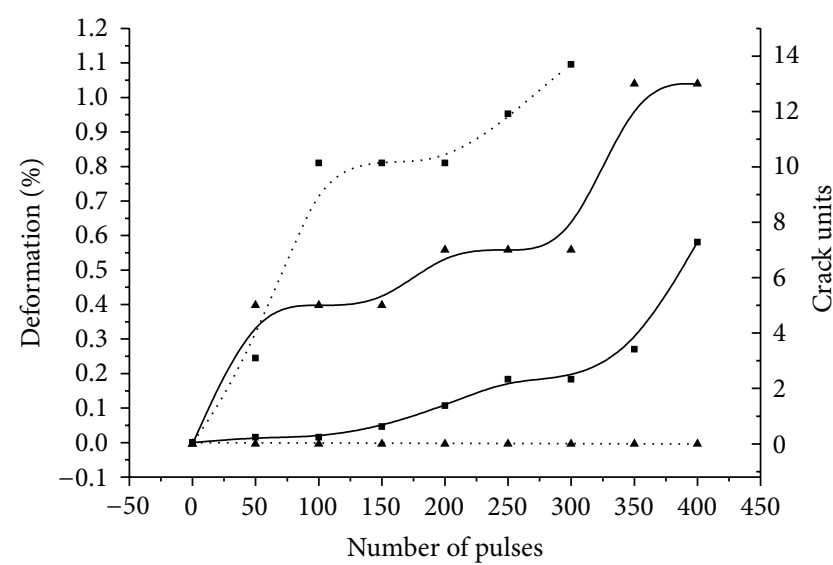

$$
\begin{array}{ll}
\text { Deformation of PS } & \text { Crack of PS } \\
-193 \mathrm{~nm} & -193 \mathrm{~nm} \\
\ldots \ldots 248 \mathrm{~nm} & \ldots \ldots 248 \mathrm{~nm}
\end{array}
$$

FIGURE 3: Defect formation versus number of pulses of neat PS at $193,248 \mathrm{~nm}$.

accordance with the polymer theory regarding the increase of mechanical resistance to be relative to the increase of molecular weight. The difference between the neat solid sample and the film on window where the later exhibited increase in dot-like defect formation with additional generation of crack-like defect is worth noticing. In latter case, the PMMA films on window and the $1900 \mathrm{Mw}$ sample had a $1 \%$ more defect growth. According to the above results besides the importance of molecular weight, there is strong indication of another critical parameter introduced by the interface in contrast to the neat sample. It is assumed that solid bulk material due to lack of interfaces shows higher mechanical resistance with stress wave energy decreasing by successive reflections until being diminished.

3.2. Study on Neat Polymer at $248 \mathrm{~nm}$. Irradiation of neat PMMA $(\mathrm{Mw}=1900 \mathrm{u})$ resulted in deformation area of $2.3 \%$ at 200 pulses whereas for $\mathrm{Mw}=120.000 \mathrm{u}$ at 200 pulses it resulted in deformation area of $0.09 \%$ with additional cracklike formation of 4.5 unit cracks.

Irradiation of neat PS $(\mathrm{Mw}=1180 \mathrm{u})$ resulted in deformation area of $0.8 \%$. In Figures 3 and 4, the results of the defect formation of the neat polymers are shown and one could observe that the PMMA generates few defects of both morphologies at $248 \mathrm{~nm}$ (compared with Figure 2), whereas PS generates more deformation of dot-like defects but less deformation of crack-like defects. These results introduce an important parameter related to the initial conditions of the sample. The preexistence of discontinuities is a random variable influencing in diverse degree the observed results as can be seen for the PMMA at $248 \mathrm{~nm}$ (Figure 4) where the sudden increase in defect formation at 150 pulses is due to preexistent discontinuity around the ablation spot recorded by holography. In most of the cases, the dot-like formation is spread all over the sample with constant size; instead, in the later example, the local high concentration of the defects 


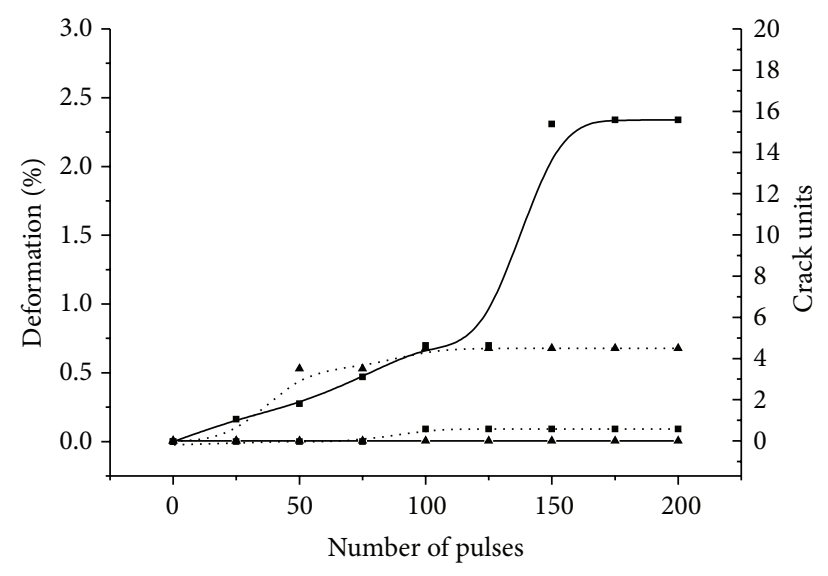

$\begin{array}{ll}\text { Deformation at } 248 \mathrm{~nm} & \text { Crack at } 248 \mathrm{~nm} \\ \ldots \text { B } & \longrightarrow \text { D } \\ \ldots \text { B } & \ldots \ldots \text { D }\end{array}$

FIgURE 4: Defect formation versus number of pulses of PMMA at $248 \mathrm{~nm}$.

represents the influence of preexistent discontinuity that allowed nucleation of defect formation. It should be noted that the graphs illustrate the evolution of defect as a function of number of pulses but the additional information on defect spatial distribution and geometrical characteristics in terms of size, shape, and propagation are provided in holograms and are used to clarify diverse behaviors.

3.3. Study on Blend Polymer at $193 \mathrm{~nm}$. Assuming that the results of neat polymer are not simulating the realistic conditions, for example, tissue surgery and varnish removal, a complementary approach was envisaged in order to achieve correspondence to real applications. Since a homogeneous material is bonded stronger than heterogeneous material and hence shows higher mechanical resistance to the laser induced deformation, a combination of polymers was used to simulate realistic conditions. The polymers used were PMMA and PS, which have different absorption coefficients at 193 and $248 \mathrm{~nm}$. The mixture was constituted from the polymers in different concentrations in order to achieve changes in absorption coefficient and efficient bond breaking due to induced phase separation.

A thin film of medium concentration of $50: 50$ PMMA : PS of high molecular weights $\mathrm{Mw}_{\text {PMMA }}=120.000 \mathrm{u}$ and $\mathrm{Mw}_{\mathrm{PS}}=240.000 \mathrm{u}$ was constructed and irradiated up to 200 pulses without traces of defect formation. The same blend polymer was then spread onto a suprasil window and irradiated with 200 pulses and again there was no trace of defect formation. The results indicate high stress resistance, which can be affected by changing the parameters both of molecular weight and of concentration. A sample on suprasil window was constructed as above with same concentration but with polymers of low molecular weight $\mathrm{Mw}_{\text {PMMA }}=1900 \mathrm{u}$ and $\mathrm{Mw}_{\mathrm{PS}}=1180 \mathrm{u}$ was irradiated with 200 pulses resulting in $0.9 \%$ deformation and 16 crack units. The influence of the molecular weight is becoming apparent with mechanical resistance increasing by increasing

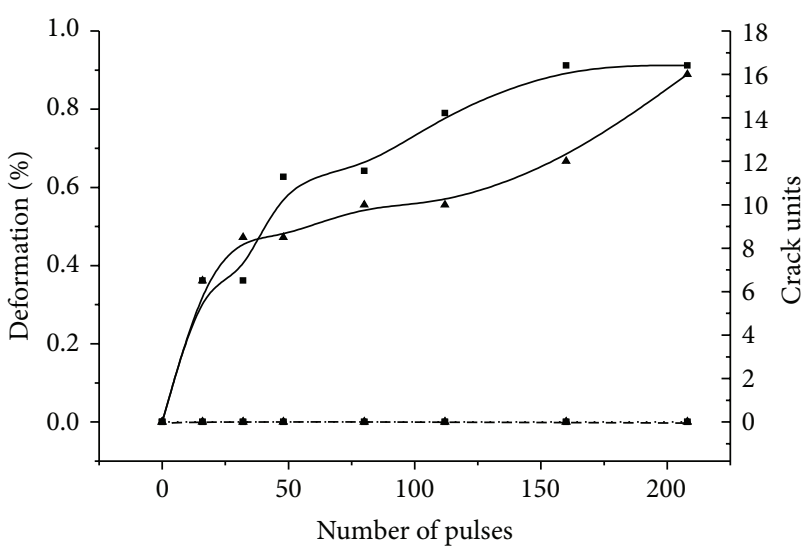

$\begin{array}{ll}\text { Deformation at } 193 \mathrm{~nm} & \text { Crack at } 193 \mathrm{~nm} \\ -- \text { B } & - \text { C } \\ -=- \text { B } & -\leftarrow \text { C } \\ \cdots \text { B } & \cdots \backsim \text { C }\end{array}$

FIGURE 5: Defect formation versus number of pulses of $50: 50$ polymer blends at $193 \mathrm{~nm}$ with different MW.

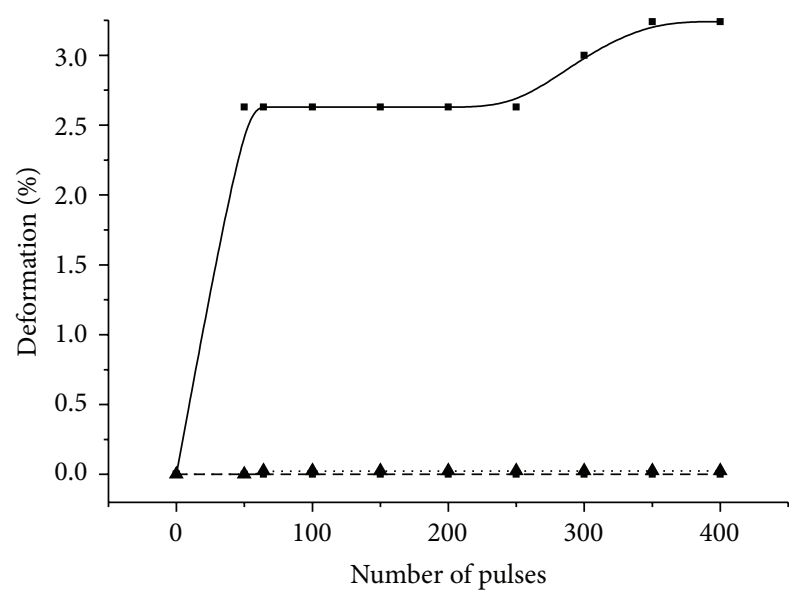

Deformation at $193 \mathrm{~nm}$

$\rightarrow-(20 \mathrm{PS} / 80 \mathrm{PMMA}$ LMW)

.... (20PS/80PMMA HMW)

- ^ - (80PS/20PMMA LMW)

FIGURE 6: Defect formation versus number of pulses of $20: 80$ and $80: 20$ blend PS : PMMA at $193 \mathrm{~nm}$.

molecular weight and can be seen in the comparison in Figure 5.

The experiment was then continued by changing the concentration of blend polymer. Two samples of same concentration of PMMA:PS as 80:20 are one for high and one for low molecular weight. The blend sample of high molecular weight $\left(\mathrm{PMMA}_{120.000} 80: \mathrm{PS}_{240.000} 20\right)$ after 200 pulses did not exhibit any deformation whereas the low molecular weight sample $\left(\mathrm{PMMA}_{1900} 80: \mathrm{PS}_{1180} 20\right)$ after 200 pulses has exhibited $2.63 \%$ and at 450 pulses $3.24 \%$. The results confirmed strong influence of molecular weight with defect formation increasing by decreasing the molecular weight. Also the results in comparison with the polymer 


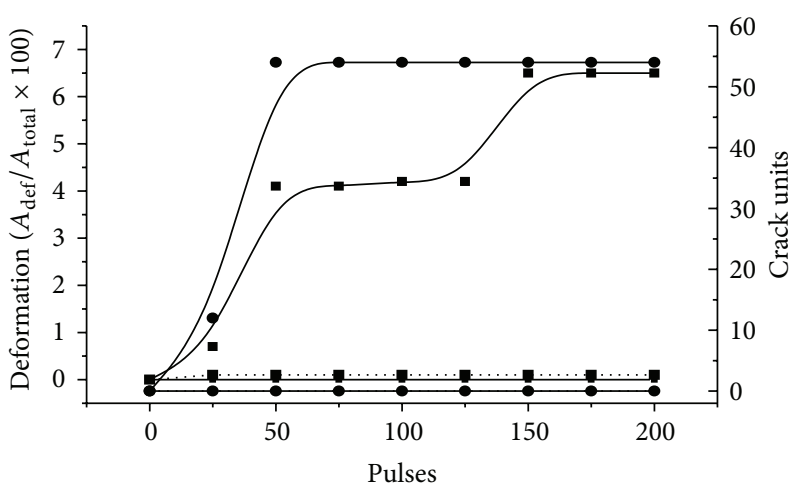

Deformation:

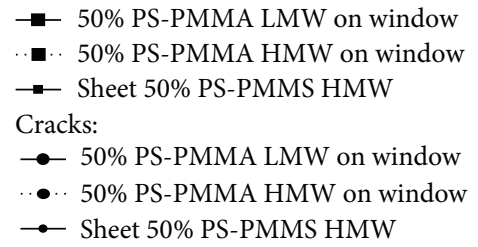

FIGURE 7: Defect formation versus number of pulses for $50: 50$ PMMA : PS in $248 \mathrm{~nm}$.

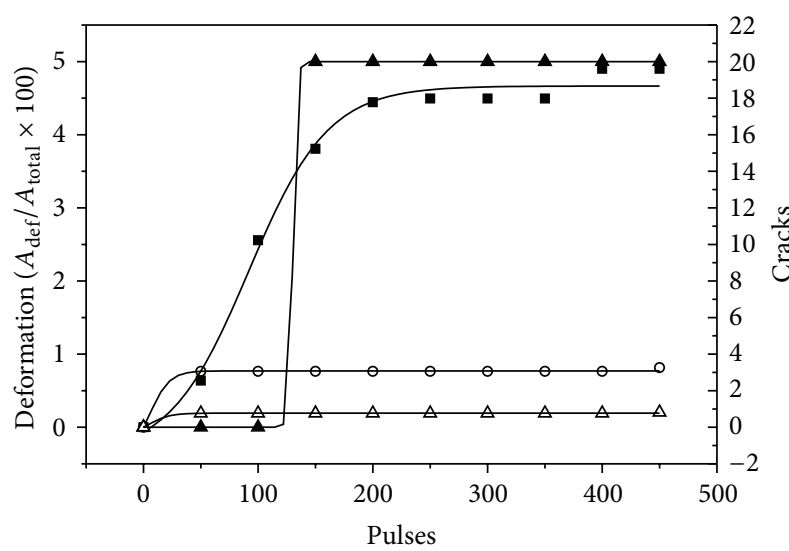

$\begin{array}{ll}\begin{array}{c}\text { 20PS-80PMMA } \\ \text { Islands }\end{array} & \text { Cracks } \\ \text { - Low M.W. } & \text { Low M.W. } \\ \text { - High M.W. } & \Delta \text { High M.W. }\end{array}$

Figure 8: Defect formation versus number of pulses in blend polymer PMMA:PS 80:20 for high and low molecular weight in $248 \mathrm{~nm}$.

concentration at 50:50 indicate higher defect formation in lower concentration of PS (Figure 6).

Therefore, by keeping low molecular weight, the experiments were continued with investigation of higher concentration of PS expecting less defect formation. Indeed, a low value of deformation of $0.05 \%$ was exhibited by the sample constructed with high concentration of PS (PMMA : PS at $20: 80$ ). Instead, higher concentrations of PMMA resulted at higher defect formation as is also illustrated in Figure 6.

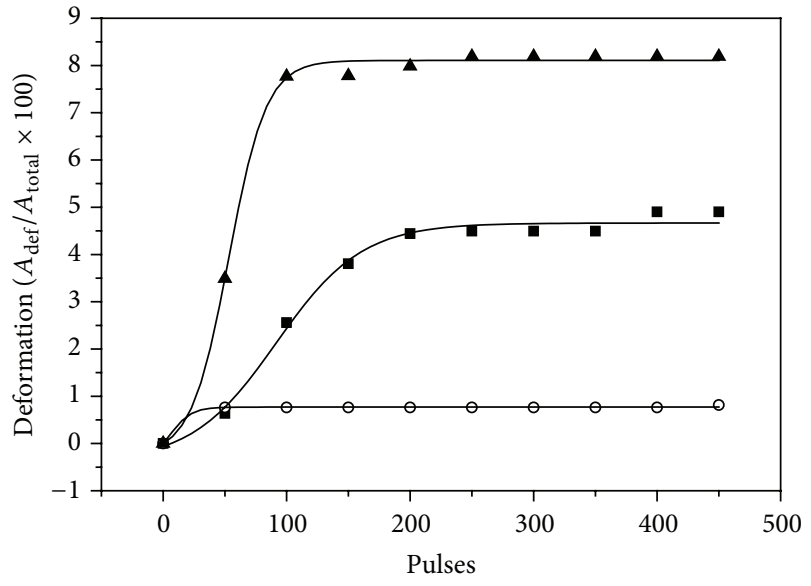

- 80PMMA-20PS low M.W.

O 80PMMA-20PS high M.W.

- 20PMMA-80PS low M.W.

(a)

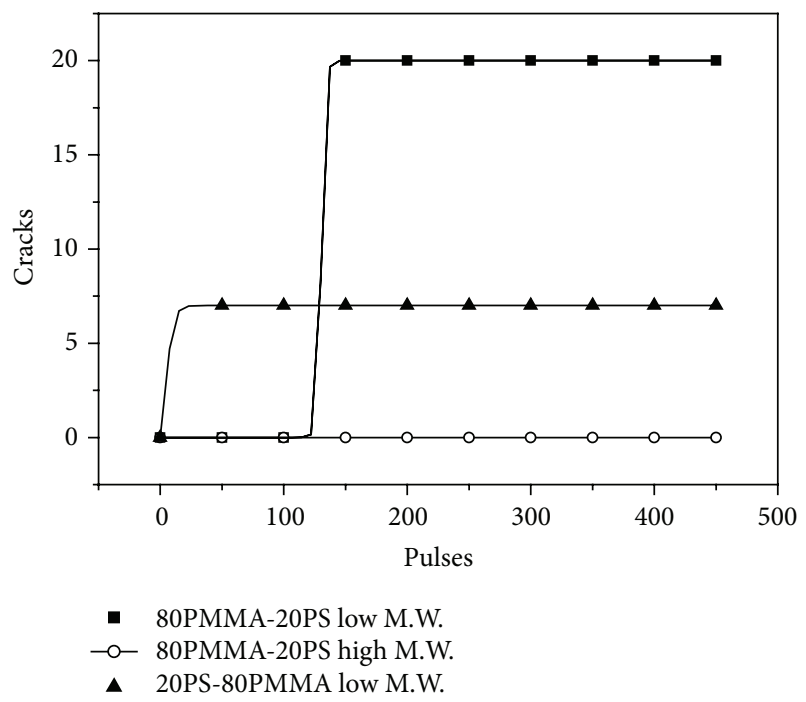

(b)

Figure 9: (a) Deformation versus number of pulses of $20: 80$ and $80: 20$ blend PS:PMMA at $248 \mathrm{~nm}$ presented two defect morphologies, in (a) the dot-like deformation. (b) The cracks reach a threshold by abrupt increase.

3.4. Study on Blend Polymer at $248 \mathrm{~nm}$. The effect of laser wavelength was investigated by performing experiments on the same samples at $248 \mathrm{~nm}$. Summarised results follow. The 50:50 PMMA : PS of high molecular weights $\mathrm{Mw}_{\mathrm{PMMA}}=$ $120.000 \mathrm{u}$ and $\mathrm{Mw}_{\mathrm{PS}}=240.000 \mathrm{u}$ was constructed and irradiated up to 200 pulses and no traces of deformation were exhibited. The same blend polymer was then spread onto a suprasil window irradiated with same number of pulses and resulted in only $0.1 \%$ deformation. The next blend polymer was 50:50 PMMA:PS with low $\mathrm{Mw}_{\text {PMMA }}=1900$ and $\mathrm{Mw}_{\mathrm{PS}}=1180$ and resulted in much higher defect formation 


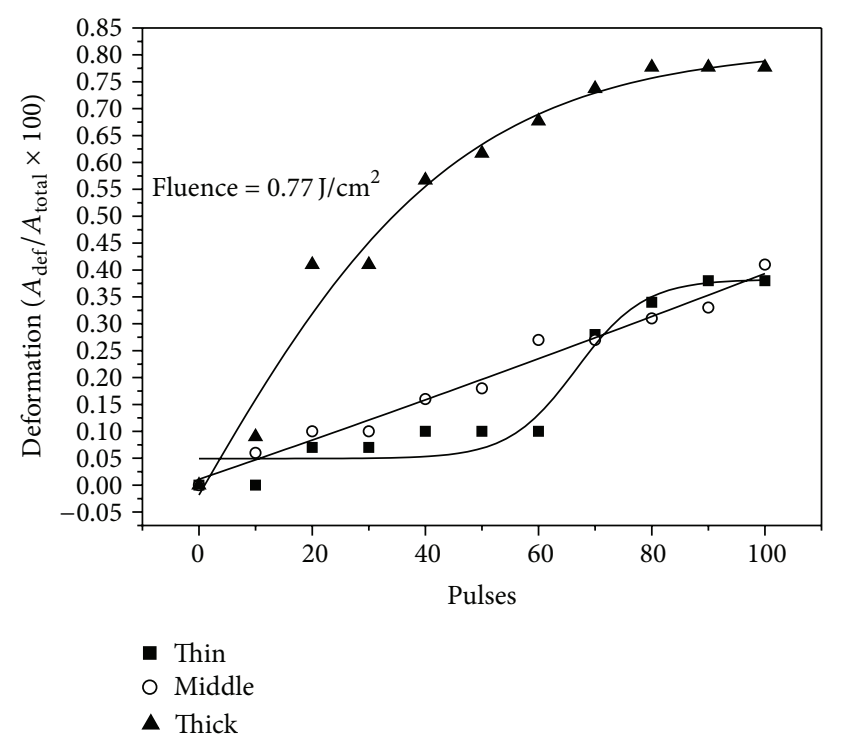

FIGURE 10: The same blend sample in various film thicknesses. It is seen that the thicker the sample, the more the defect formation.

as compared to $193 \mathrm{~nm}$, that is, $4.6 \%$ deformation and 54 crack units. The results are seen in Figure 7.

In the following, two samples of $80: 20$ concentration of PMMA : PS were constructed, one with high and one with low molecular weight. The blend sample of high molecular weight $\left(\mathrm{PMMA}_{120.000} 80: \mathrm{PS}_{240.000} 20\right)$ after 200 pulses exhibited $0.76 \%$ and increased after 450 pulses to $0.8 \%$ deformation value. The result is in contrast to the ones obtained with $193 \mathrm{~nm}$ irradiation where the same blend was intact. The low molecular weight sample $\left(\mathrm{PMMA}_{1900} 80: \mathrm{PS}_{1180} 20\right)$ after 200 pulses exhibited $4.44 \%$ and 20 crack units and at 450 pulses increased to $4.9 \%$ while the crack units did not increase. Again, the results showed increase in defect formation by decreasing the molecular weight. Also in comparison with the polymer concentration at $50: 50$, the results indicate again higher defect formation in lower concentration of PS.

Therefore, the next sample was of low molecular weight and with higher concentration of PS, so PMMA 20 : PS 80. At 200 pulses, $7.98 \%$ deformation and 7 crack units were exhibited with slight increase up to $8 \%$ at 400 pulses. The results of the two blend polymers with different concentrations in $248 \mathrm{~nm}$ do not exhibit the straightforward relation of higher defect formation in lower concentration of PS as compared to the results at $193 \mathrm{~nm}$. This is due to the combination of the two defect morphologies that are apparent in $248 \mathrm{~nm}$ while at $193 \mathrm{~nm}$ were generated only dot-like structures. Hence, generation of two defect morphologies in all samples was observed in $248 \mathrm{~nm}$ as it is shown in the graphs of Figures 8 and 9. It is noted that both defect formations reach a threshold, but by following different paths, the crack-like defect formation follows an abrupt increase whereas the dotlike follows a gradual one.

3.5. Study on Doped PMMA-NapH at $193 \mathrm{~nm}$. The critical importance of absorption coefficient was investigated

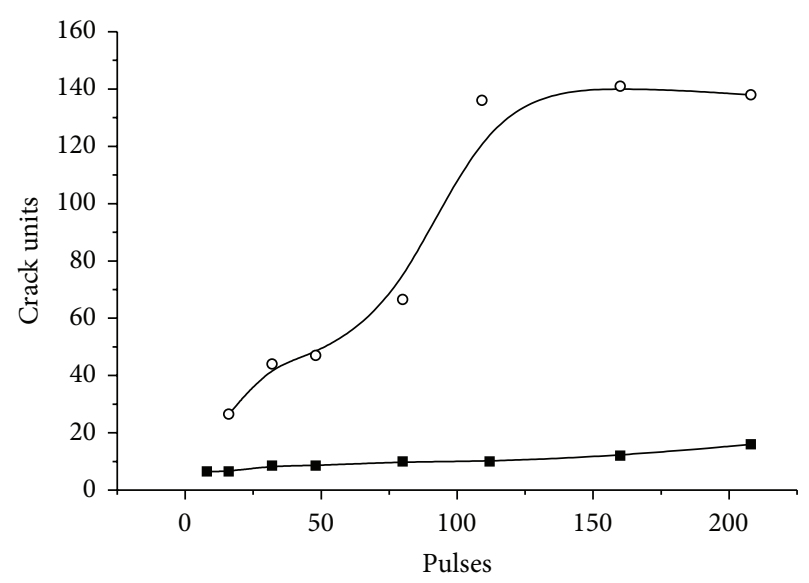

50\% PS-PMMA LMW

Cracks:

$\rightarrow 24$ hours after the preparation

$\rightarrow$ - One week after the preparation

(a)

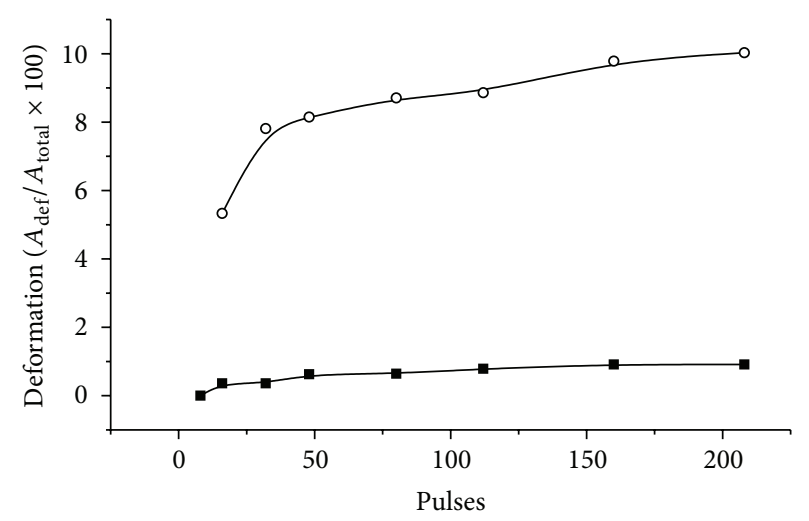

50\% PS-PMMA HMW on window Deformation:

- 24 hours after the preparation

- - One week after the preparation

(b)

FIGURE 11: (a) Comparison between two different aging conditions, new and one-week aged samples, shows preconditioned samples generating higher defect formation (here the morphology shown represents cracks). (b) The same comparison with the shown defect morphology of dot-like.

by changing the concentration of the absorbing PS in the PMMA/PS blend. By changing the concentration in blends, modifications are induced both in optical and in mechanical properties of the material. In order to exclude modification in mechanical properties but induce optical modification due to change in absorption coefficient, an experiment was performed on doped with $1 \%$ naphthalene PMMA. The results showed $1 \%$ deformation of dot-like structures after 200 pulses that is the same deformation for neat PMMA but a 60\% decrease in crack-like defects (5 crack units). Therefore, the total deformation tends to 


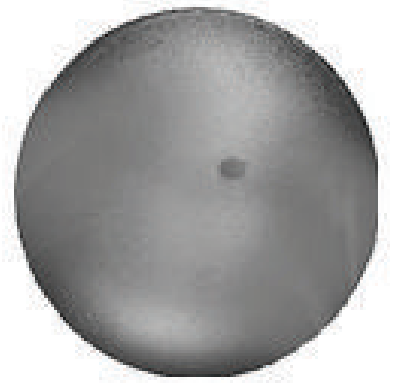

5 pulses

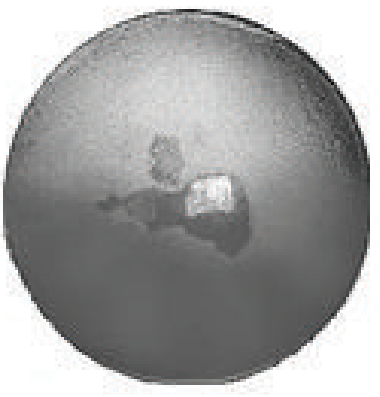

50 pulses

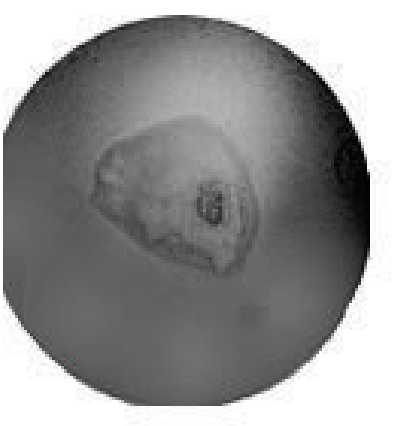

125 pulses

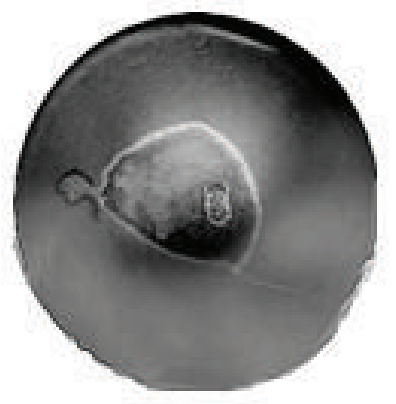

170 pulses

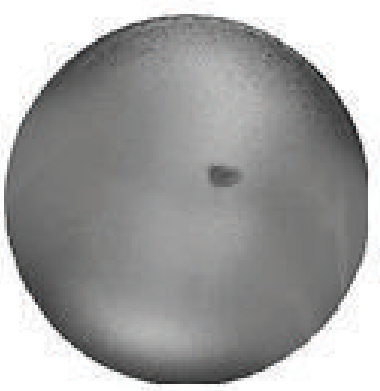

15 pulses

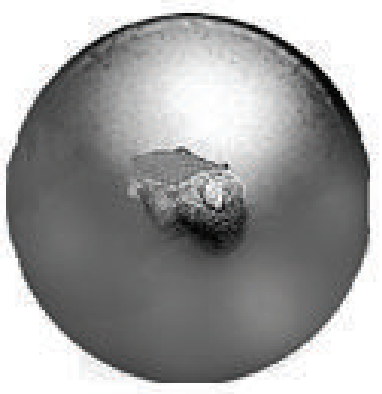

80 pulses

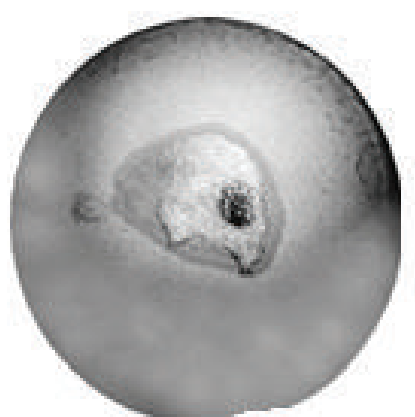

130 pulses

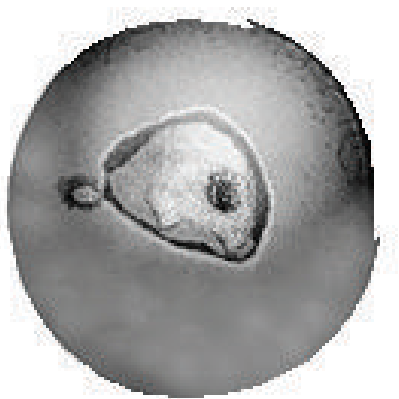

200 pulses

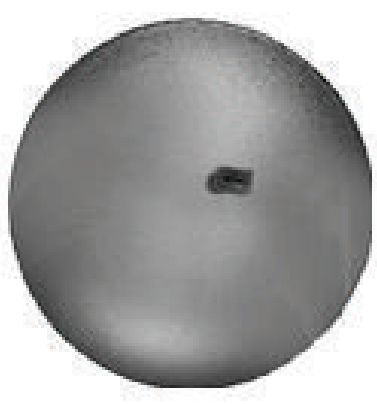

30 pulses

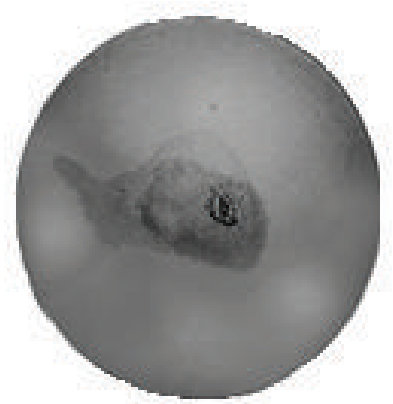

120 pulses
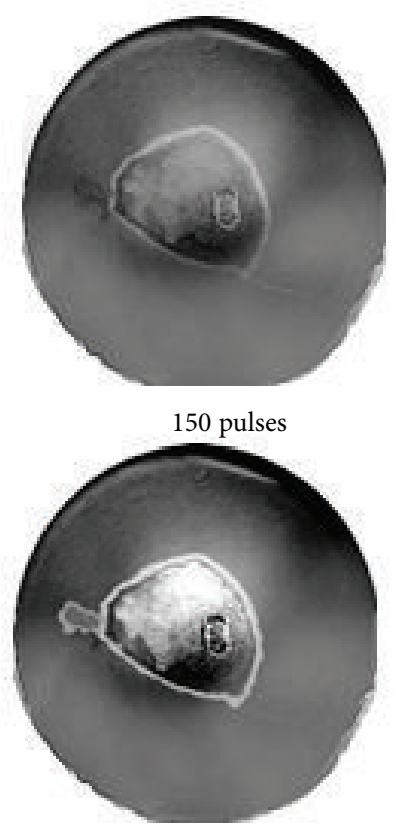

240 pulses

FIGURE 12: Defect formation during the pulse accumulation process.

be considerably decreased in PMMA doped with a highly absorbing molecule. If we compare the defect formation for Naph doped PMMA with the high absorbing PS, we can conclude the importance of mechanical properties, which prove to be equally critical as that of the optical ones.
3.6. Study on Film Thickness at $193 \mathrm{~nm}$. The influence of film thickness was studied with construction of three blend samples of PMMA 80 : PS 20 spread as film on suprasil with thickness starting from 30 to $70 \mu \mathrm{m}$. The defect formation was increasing as the thickness was increasing, so for the thin sample the total deformation was $1.93 \%$, for the medium 


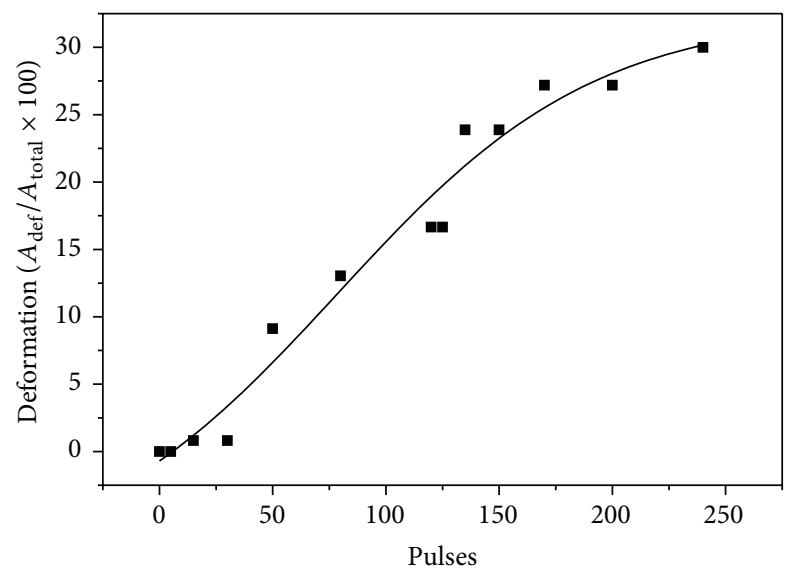

FIGURE 13: The graph of blend of Figure 12, a PMMA 80 : PS 20 irradiated at $193 \mathrm{~nm}$.

sample was $2.08 \%$, and for the thick sample was $3.95 \%$, as can be seen in the graph in Figure 10. It is noted that no crack formation was exhibited in consistence with the previous results of PMMA 80 :PS 20 at $193 \mathrm{~nm}$ and opposed to the result of the same blend at $248 \mathrm{~nm}$ where crack formation was strong.

3.7. Study on Aged and Preconditioned Samples in $193 \mathrm{~nm}$. All the above experiments were performed just after the drying of the polymer solution, which is completely dry after a period of 24 hours. Further experiments were performed with old polymer powder and the prepared samples left in ambient temperature and relative humidity for one week (168 hours).

A neat film on suprasil window of low molecular weight PMMA was irradiated after a week and $1.53 \%$ value of deformation with 29 crack units was exhibited at 200 pulses. Both values compared to the results of the newly constructed sample are much higher. The blend PMMA 50 : PS 50 of low molecular weight at the same number of pulses exhibited a $10 \%$ defect formation and 138 crack units (Figure 15). The defect formation is considerable and the comparison between new and aged samples is shown in Figures 11(a) and 11(b).

Polymer samples of PMMA 80: PS 20 of low molecular weight were also constructed with old polymer powder and $30 \%-50 \%$ deformation was generated. The graph is in Figure 13 and the holograms are shown in Figure 12.

Another interesting observation derives from the fact that in polymers constructed with the old powder no cracks were exhibited instead spread island/dot-like deformations cover big part of the sample. This is attributed to the factor of adhesion strength.

The amount of deformation exhibited in the old and preconditioned polymer samples introduces more parameters equally critical as the molecular weight, concentration, and wavelength since the highest deformation values were observed. By observing the holograms shown in the pictures (Figures 12 and 14), the defect morphology resembles detachments of the underlying layer that by pulse accumulation are growing and expanding.

\section{Discussion}

Figures show series of recordings of solid/blend polymers while being under excimer irradiation. Various parameters in the preparation and construction of the samples were investigated. Each parameter contributed a significant role in the unfolding of structural deformations and they are critical indicators of mechanical resistance to the photoablative effect. The examination on the samples and parameters shown in this reported experimental synopsis reveals that there is strong effect induced in delocalized from the ablation spot extent of sample. Deformation rises with accumulation of pulses with the first 30 pulses having no detectable influence except in the ablation spot. During the next 20 pulses, an extended detachment occurs surrounding the ablation spot and expanding outwards. The effect can be attributed to local dewetting due to loose adherence of the blend polymer film on the suprasil window. The most expected deformation feature would be in the form of crack after the yield point of the blend polymer would have resulted in fracture. Since the system is a spread polymer film $(\approx 150 \mu \mathrm{m}$ thickness) on quartz window, an unstable thermodynamic state can be assumed tending to detach off and fold in order to acquire the maximum thermodynamic equilibrium. The deformation effect is cumulative and the integrity of the sample is deteriorating in each recording. The maximum deformation is exhibited though from 50 to 125 pulses with the last 115 pulses reaching almost a plateau.

The above relevance is questionable since in polymer film on suprasil window more structural morphologies were observed than detachments attributed to dewetting. More results reveal formation of dot-like and bubble-like or isle-like local discontinuities. The experimental observation indicates variability in morphologies which cannot be attributed to the thermodynamic instability of the polymer-window system. The crack-like or dendrite-like patterns were observed and attributed to strong cohesion. High degree of bifurcation in dendrite-type defects is shown in sample of lower cohesion as in fracture dynamics in which the deposition speed of 


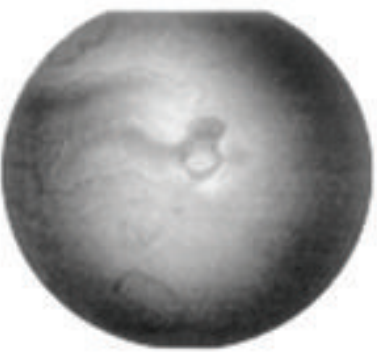

5 pulses

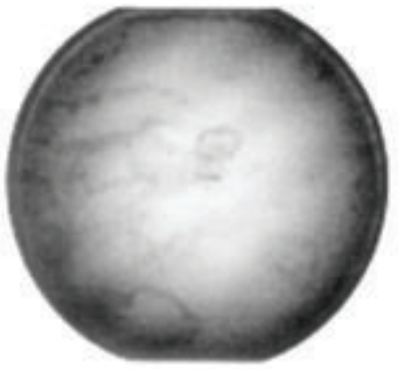

50 pulses

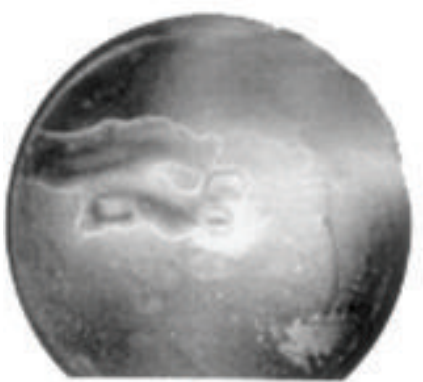

125 pulses

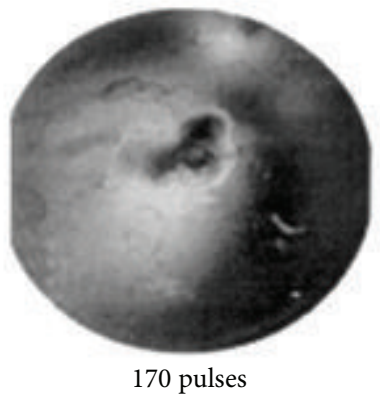

170 pulses

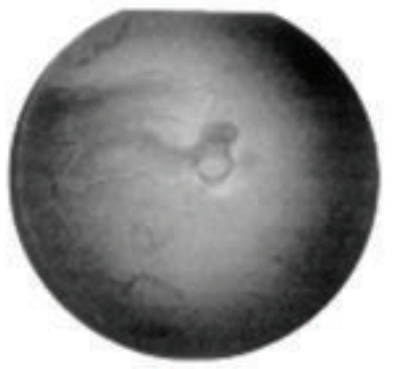

15 pulses

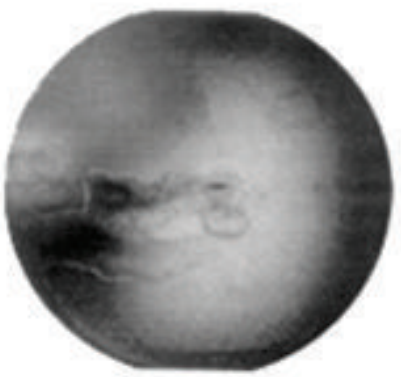

80 pulses

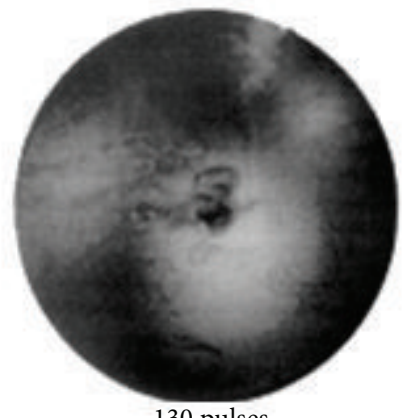

130 pulses

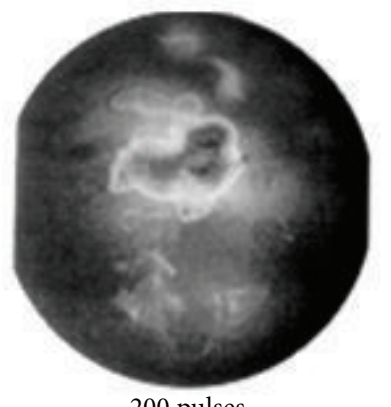

200 pulses

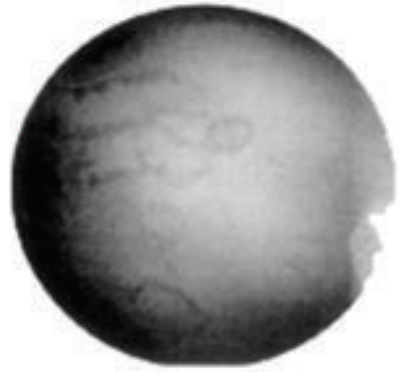

30 pulses

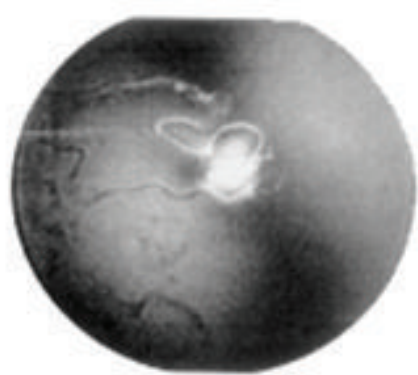

120 pulses

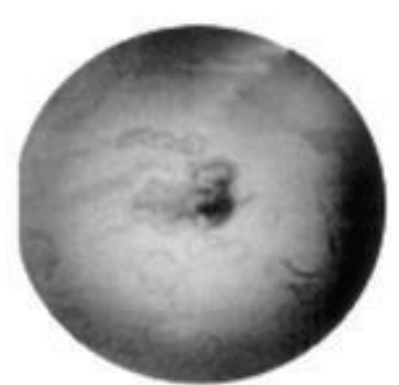

150 pulses

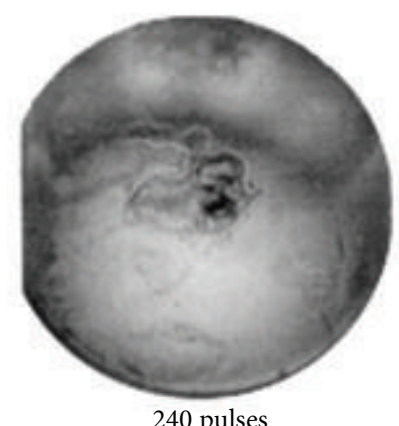

240 pulses

FIGURE 14: Defect formation during the pulse accumulation process.

energy is greater than the maximum speed propagation of the crack (Rayleigh). The existence of interfaces and the degree of adhesion strength are expressed as parameters of prime importance.

We have examined some dominant parameters which could contribute to the structural and mechanical disintegration of ablated surfaces with preliminary results being consistent. The mechanisms involved in photoablation processes were investigated leading to the inhomogeneity of materials as a leading mechanical parameter for deformation occurrence. The results are in accordance with a number of different studies performed on the field of laser ablation under different experimental techniques and experimental aims [18, 25-35]. 


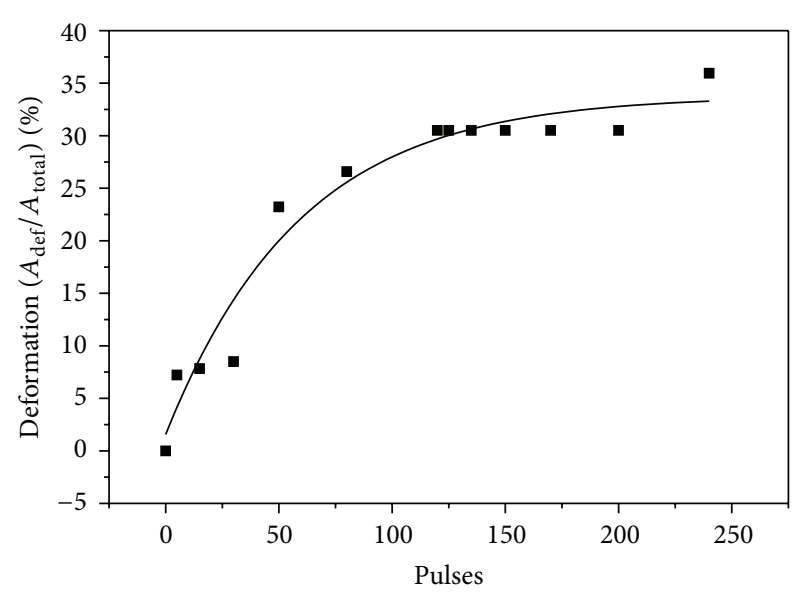

FIgURE 15: The profile graph of Figure 14.

\section{Appendix}

The holograms and interferograms were recorded on photosensitive plates, which are produced by HRT Holographic Recording Technologies GmbH model BB-640 red sensitive. follows.

The rehalogenating process for the development is as

(1) Developer $\left(1000 \mathrm{~cm}^{3}\right)$ :

sodium carbonate, anhydrous $70 \mathrm{~g}$,

sodium hydroxide $15 \mathrm{~g}$,

metol (4-(methylamino)-phenol-sulfate) $4 \mathrm{~g}$,

ascorbic acid $25 \mathrm{~g}$,

developing to a density of 2.5 .

(2) Stop bath $\left(1000 \mathrm{~cm}^{3}\right)$ :

sodium hydrogen sulfate crystals $5 \mathrm{~g}$,

rinsing briefly in cold water and then bleaching.

(3) Bleach $\left(1000 \mathrm{~cm}^{3}\right)$ :

copper sulfate (pentahydrate) $25 \mathrm{~g}$,

potassium bromide $100 \mathrm{~g}$,

sodium hydrogen sulfate crystals $5 \mathrm{~g}$,

bleaching until the hologram is clear and then rinsing in cold water.

\section{Conflict of Interests}

The author declares that there is no conflict of interests regarding the publication of this paper.

\section{Acknowledgments}

The ongoing work has been supported by the Improving Human Potential Program DGXII (HPRI-CT-1999-00074), by the Marie Curie Training Site Program, by the TMR Programme (no. ERB FMRXCT98-0188), and by PENED
HOLOTECH proposal administered by the Greek Ministry of Development and it is continuously supported by the ULF Program of IESL/FORTH. The above research is part of several studies and students Antonia Bonarou, M.S., and Laura Antonucci, Ph.D., are greatly acknowledged for their contribution in execution of experimental work. The author through the publication of these results wishes to attribute a special memorial to colleague Dr. Savas Georgiou with whom the author has been closely working on this topic and his sudden death has deprived everyone from an excellent scientist and coworker, devoted researcher and a good friend.

\section{References}

[1] D. Bäuerle, Laser Processing and Chemistry, Springer, Berlin, Germany, 2000.

[2] D. Bauerle, Laser Processing and Chemistry, Springer, Berlin, Germany, 3rd edition, 2011.

[3] D. J. Krajnovich and J. E. Xazquez, "Formation of "intrinsic" surface defects during $248 \mathrm{~nm}$ photoablation of polyimide," Journal of Applied Physics, vol. 73, p. 3001, 1993.

[4] J.-F. Silvain, H. Niino, S. Ono, S. Nakaoka, and A. Yabe, "Surface modification of elastomer/carbon composite by Nd+:YAG laser and KrF excimer laser ablation," Applied Surface Science, vol. 141, no. 1-2, pp. 25-34, 1999.

[5] B. Hopp, M. Csete, K. Revesz, J. Vinko, and Z. Bor, "Formation of the surface structure of polyethylene-terephtalate (PET) due to ArF excimer laser ablation," Applied Surface Science, vol. 96, pp. 611-616, 1996.

[6] F. Wagner and P. Hoffmann, "Structure formation in excimer laser ablation of stretched poly(ethylene therephthalate) (PET): the influence of scanning ablation," Applied Physics A, vol. 69, no. 1, supplement, pp. S841-S844, 1999.

[7] J. Heitz, E. Arenholz, D. Bäuerle, H. Hibst, A. Hagemeyer, and G. Cox, "Dendritic surface structures on excimer-laser irradiated PET foils," Applied Physics A Solids and Surfaces, vol. 56, no. 4, pp. 329-333, 1993.

[8] S. Watanabe, T. J. Flotte, D. J. McAuliffe, and S. L. Jacques, "Putative photoacoustic damage in skin induced by pulsed ArF excimer laser," Journal of Investigative Dermatology, vol. 90, no. 5, pp. 761-766, 1988.

[9] A. G. Doukas, D. J. McAuliffe, and T. J. Flotte, "Biological effects of laser-induced shock waves: structural and functional cell damage in vitro," Ultrasound in Medicine and Biology, vol. 19, no. 2, pp. 137-146, 1993.

[10] in 9th annual Laser-Tissue Interaction Conference, S. L. Jacques, Ed., vol. 3254 of Proceedings of SPIE, Washington, DC, USA, 1998.

[11] C. B. Gabrieli, E. Pacella, S. Abdolrahimzadeh, F. Regine, and R. Mollo, "Excimer laser photorefractive keratectomy for high myopia and myopic astigmatism," Ophthalmic Surgery and Lasers, vol. 30, no. 6, pp. 442-448, 1999.

[12] R. A. Lindley, R. M. Gilgenbach, C. H. Ching, J. S. Lash, and G. L. Doll, "Resonant holographic interferometry measurements of laser ablation plumes in vacuum, gas, and plasma environments," Journal of Applied Physics, vol. 76, no. 9, pp. 5457-5472, 1994.

[13] S. Georgiou, V. Zafiropulos, D. Anglos, C. Balas, V. Tornari, and C. Fotakis, "Excimer laser restoration of painted artworks: procedures, mechanisms and effects," Applied Surface Science, vol. 127-129, pp. 738-745, 1998. 
[14] V. Tornari, D. Fantidou, V. Zafiropulos, N. A. Vainos, and C. Fotakis, "Photomechanical effects of laser cleaning: a long-term non-destructive holographic interferometric investigation on painted artworks," in 3rd International Conference on: Vibration Measurements by Laser Techniques: Advances and Applications, vol. 3411 of Proceedings of SPIE, pp. 420-430, June 1998.

[15] I.-Y. S. Lee, X. Wen, W. A. Tolbert, D. D. Dlott, M. Doxtader, and D. R. Arnold, "Direct measurement of polymer temperature during laser ablation using a molecular thermometer," Journal of Applied Physics, vol. 72, no. 6, pp. 2440-2448, 1992.

[16] A. D. Zweig, V. Venugopalan, and T. F. Deutsch, "Stress generated in polyimide by excimer-laser irradiation," Journal of Applied Physics, vol. 74, no. 6, pp. 4181-4189, 1993.

[17] S. Siano, R. Pini, and R. Salimbeni, "Variable energy blast modeling of the stress generation associated with laser ablation," Applied Physics Letters, vol. 74, no. 9, pp. 1233-1235, 1999.

[18] K. J. Gåsvik, Optical Metrology, Wiley, New York, NY, USA, 2nd edition, 1995.

[19] C. H. Vest, Holographic Interferometry, Academic, New York, NY, USA, 1971.

[20] R. A. Lindley, R. M. Gilgenbach, and C. H. Ching, "Resonant holographic interferometry of laser-ablation plumes," Applied Physics Letters, vol. 63, no. 7, pp. 888-890, 1993.

[21] M. Olfert and W. W. Duley, "Holographic interferometry of isotherms during laser drilling of fused quartz," Journal of Physics D: Applied Physics, vol. 29, no. 5, pp. 1140-1145, 1996.

[22] A. Kreuttner, B. Lau, A. Mann et al., "Holographic interferometry for the display of shock wave induced deformations and vibrations-A contribution to laser lithotripsy," Lasers in Medical Science, vol. 8, no. 3, pp. 211-220, 1993.

[23] I.-Y. S. Lee, W. A. Tolbert, D. D. Dlott et al., Journal of Imaging Science and Technology, vol. 36, p. 180, 1992.

[24] F. C. Burns and S. R. Cain, "The effect of pulse repetition rate on laser ablation of polyimide and polymethylmethacrylate-based polymers," Journal of Physics D: Applied Physics, vol. 29, no. 5, pp. 1349-1355, 1996.

[25] W. D. Callister, Materials Science and Engineering: An Introduction, John Wiley \& Sons, New York, NY, USA, 1997.

[26] J. W. Smith, E. J. Kramer, and P. J. Mills, "Tailored adhesion at polymer/nonpolymer interfaces," Journal of Polymer Science, Part B: Polymer Physics, vol. 32, no. 10, pp. 1731-1744, 1994.

[27] M. Lokhandwalla and B. Sturtevant, "Fracture mechanics model of stone comminution in ESWL and implications for tissue damage," Physics in Medicine and Biology, vol. 45, no. 7, pp. 1923-1940, 2000.

[28] D. Blanchard, R. Casalengo, M. Pierre, and H. P. Trommsdorff, "Impulsive generation of hypersonic waves: an application to the measurement of acoustic properties of poly-methylmethacrylate," Journal of Applied Physics, vol. 65, no. 9, article 3351, 1989.

[29] M. Centurion, Y. Pu, Z. Liu, D. Psaltis, and T. W. Hänsch, "Holographic recording of laser-induced plasma," Optics Letters, vol. 29, no. 7, pp. 772-774, 2004.

[30] J. R. Asay, D. L. Lamberson, and A. H. Guenther, "Pressure and temperature dependence of the acoustic velocities in polymethylmethacrylate," Journal of Applied Physics, vol. 40, no. 4, pp. 1768-1783, 1969.

[31] M. Jie and C. Y. Tang, "Limitation on crack speed for a strip-craze model applied to PMMA," Theoretical and Applied Fracture Mechanics, vol. 34, no. 1, pp. 11-16, 2000.
[32] S. Wu, Polymer Interface and Adhesion, Marcel Dekker, New York, NY, USA, 1982.

[33] A. Bonarou, L. Antonucci, V. Tornari, S. Georgiou, and C. Fotakis, "Holographic interferometry for the structural diagnostics of UV laser ablation of polymer substrates," Applied Physics A: Materials Science and Processing, vol. 73, no. 5, pp. 647-651, 2001.

[34] A. Athanassiou, K. Lakiotaki, V. Tornari, S. Georgiou, and C. Fotakis, "Photocontrolled mechanical phenomena in photochromic doped polymeric systems," Applied Physics A: Materials Science and Processing, vol. 76, no. 1, pp. 97-100, 2003.

[35] V. Tornari, D. Fantidou, V. Zafiropulos, N. A. Vainos, and C. Fotakis, "Photomechanical effects of laser cleaning: a long-term non-destructive holographic interferometric investigation on painted artworks," in 3rd International Conference on: Vibration Measurements by Laser Techniques: Advances and Applications, E. P. Tomasini, Ed., vol. 3411 of Proceedings of the SPIE, pp. 420430, Ancona, Italy, June 1998. 

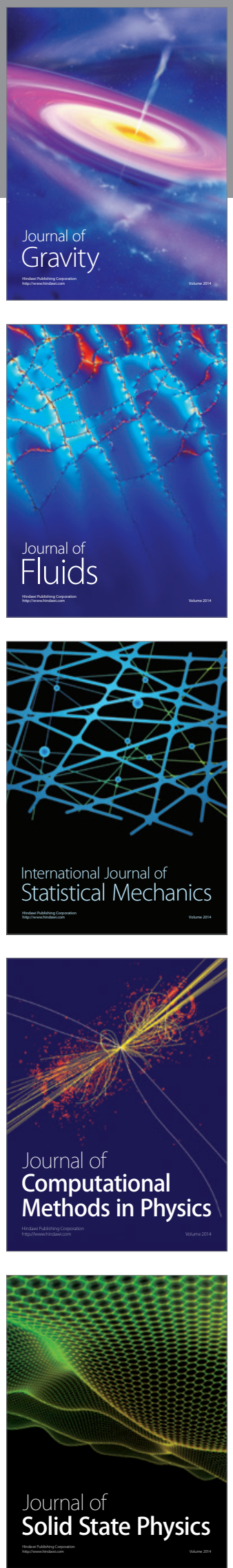

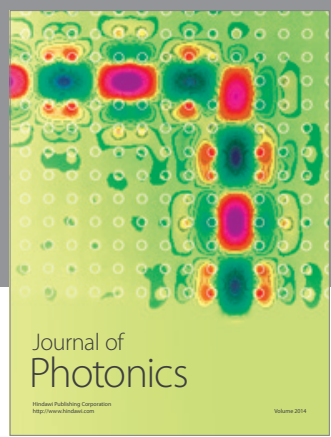

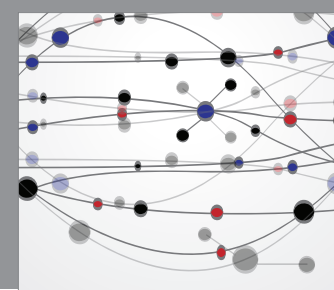

The Scientific World Journal

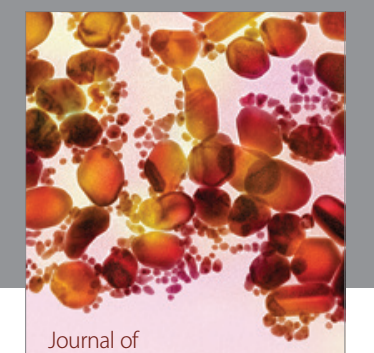

Soft Matter
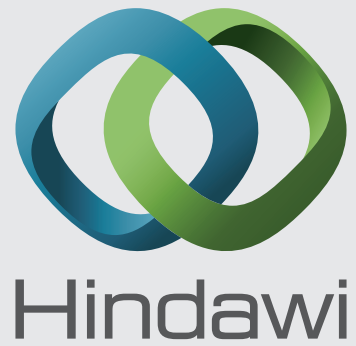

Submit your manuscripts at

http://www.hindawi.com
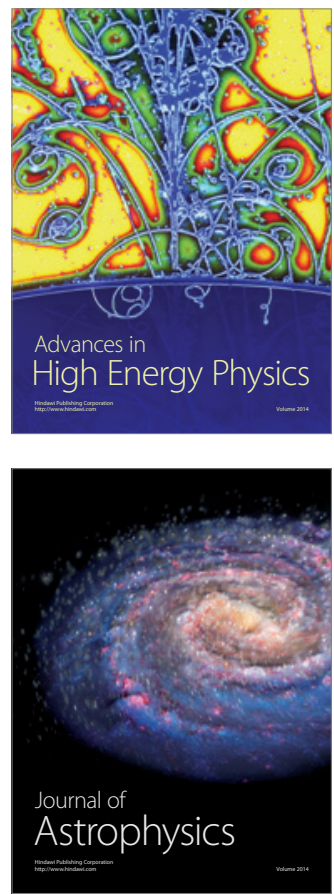
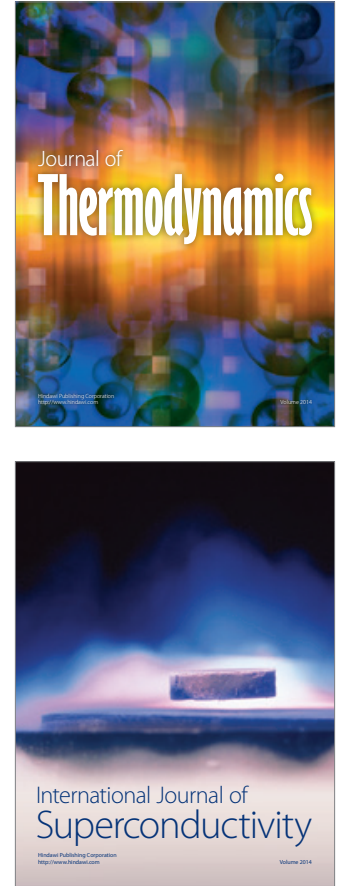
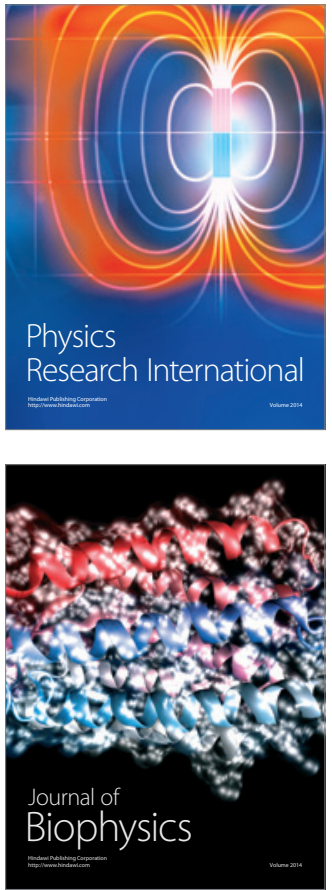
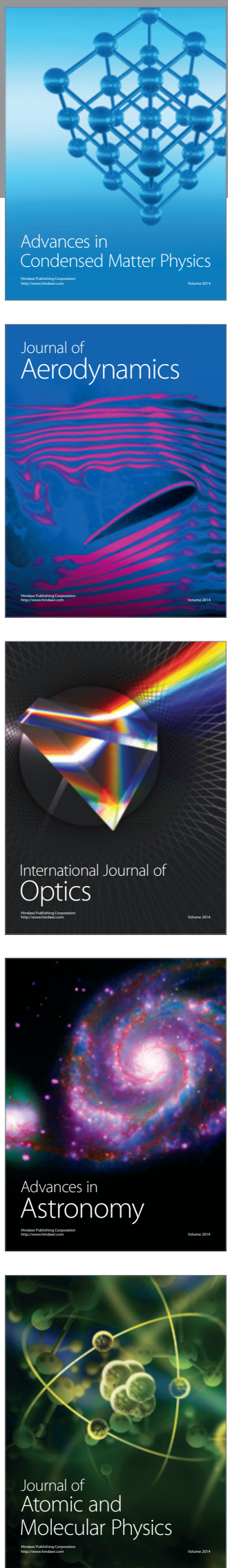